\title{
Bayesian Link Adaptation under a BLER Target
}

\author{
Vidit Saxena*† and Joakim Jaldén* \\ vidits@kth.se_jalden@kth.se \\ *KTH Royal Institute of Technology, Stockholm, Sweden \\ ${ }^{\dagger}$ Ericsson Research, Stockholm, Sweden
}

\begin{abstract}
The optimal modulation and coding scheme (MCS) for wireless transmission depends on the dynamic wireless channel state. Hence, wireless link adaptation relies on periodically reported channel quality index (CQI) values to select the optimal MCS for each transmission instance. However, to optimize link performance for a given wireless environment, current link adaptation techniques rely on tuning parameters such as a block error rate (BLER) target and algorithm adjustments that are difficult to optimize heuristically. Here, we propose BayesLA, a Bayesian link adaptation scheme that does not require careful parameter tuning for optimal link performance in diverse wireless environments. BayesLA, which is inspired by the Thompson Sampling approach widely used for online learning, efficiently learns the MCS success probabilities conditioned on the reported CQI values. Through numerical simulations for a Rayleigh fading wireless channel and a typical cellular link configuration, we demonstrate that BayesLA outperforms stateof-the-art outer loop link adaptation (OLLA) approach in terms of the realized link throughput for a given BLER target.
\end{abstract}

\section{INTRODUCTION}

Link adaptation is a critical component in the efficient operation of wireless networks. By optimally selecting the link parameters in response to the observed wireless channel state, link adaptation can significantly improve the link performance in terms of its throughput and average packet error rate. Current link adaptation techniques rely on periodically reported channel quality index (CQI) values to dynamically adjust the modulation and coding scheme (MCS) used for transmitting physical-layer transport blocks over the air [1]. Link adaptation is applicable to uplink as well as downlink transmission in wireless cellular networks. However, downlink link adaptation is substantially more challenging on account of quantized, delayed, and noisy CQI feedback from the user equipment. Hence, we address downlink link adaptation in the rest of this paper.

Link adaptation in wireless cellular networks comprises two inter-dependent control loops: and inner loop also known as adaptive modulation and coding (AMC), and outer loop link adaptation (OLLA) respectively. For a reported CQI value, AMC estimates the optimal MCS using lookup tables that are generated offline and stored at the base station. The lookup tables provide an approximate transmission success probability for each MCS conditioned on the CQI. However, owing to modeling errors in the lookup table, the realized link performance deviates from the optimal performance predicted by AMC. To compensate for the modeling errors that affect AMC performance, OLLA estimates an offset value for the AMC parameters iteratively over several transmissions. The
AMC-OLLA scheme strives to maximize the link throughput under an externally defined block error rate (BLER) target.

The AMC-OLLA approach (OLLA for brevity hereon) for link adaptation suffers from several issues. First, the lookup tables occupy substantial memory space in the resourceconstrained base station hardware. Second, the optimal BLER target, in terms of maximizing the link throughput, does not have a closed-form solution and typically varies between $10 \%$ and $30 \%$ [2], [3]. Another drawback of OLLA approach is that the step size for AMC offset adjustments is defined heuristically. Using a small step size leads to slow OLLA convergence. Conversely, using large step sizes allows OLLA converge quicker at the cost of large oscillations around the optimum. In a field study of live $4 \mathrm{G}$ networks [4], OLLA was found to have a strong impact on the network performance.

Several extensions have been proposed for OLLA which alleviate some of the drawbacks mentioned above. In [5], a supervised offline scheme was proposed to replace the AMC lookup tables with compact artificial neural network representations. In [6], a hypothesis testing framework was proposed to optimize the OLLA step size for faster convergence. The optimality of BLER target was investigated in [3], which also derived an approximate analytical expression that maximizes the throughput. A theoretically motivated online learning scheme for link adaptation was proposed in [7], which speeds up OLLA by learning from multiple parallel links.

In this paper, we propose BayesLA, a Bayesian approach for link adaptation. BayesLA models the MCS success probabilities conditioned on the CQI through a suitable probability distribution. Over successive transmissions, BayesLA updates these probability distributions based on the observed transmission success (i.e., ACK) and failure (i.e., NACK) events. BayesLA reduces the memory footprint of lookup tables used by OLLA by encoding the MCS success probabilities with a small number of parameters. Further, in contrast to OLLA, BayesLA does not strive to match the BLER target. Instead, BayesLA maximizes the expected link throughput subject to the BLER target by automatically converging to the optimal, throughputmaximizing, BLER allowed by the target value.

BayesLA is inspired by the Thompson sampling approach, which has been widely studied in machine learning and has been applied in recommender systems as well as operations research [8]. Recently, Thompson sampling has also been shown to improve the throughput of WiFi links in [9]. In contrast to the scheme in [9] that optimized for the link throughput, BayesLA optimizes link throughput subject to 
the BLER target. Our recent work in [10] proposed the first constrained Thompson sampling for this problem that provides theoretical performance guarantees compared to an oracle policy. Compared to [10], BayesLA conditions the MCS success probabilities on the CQI. Further, we incorporate the offline AMC lookup tables to significantly speed up BayesLA learning for the initial few transmissions. We conduct numerical simulations for cellular links over a Rayleigh fading channel to show that BayesLA outperforms OLLA while alleviating the dependence on heuristically tuned parameters.

\section{Model AND OBJECTIVES}

\section{A. Wireless Link Model}

In each frame indexed by $t=1, \ldots, T$ scheduled for downlink transmission, the base station packs information bits into variable-sized transport blocks to be transmitted over the physical air interface. The transport block size $D_{k(t)}$ is dictated by the choice of the MCS index $k(t) \in\{1, \ldots, K\}$ in frame $t$. The transport bits is first encoded using an errorprotection channel code and subsequently interleaved with a pseudo-random sequence in order to randomize the effect of noise and interference. Subsequently, the channel-encoded bits are mapped onto complex-valued symbols defined by the modulation order that corresponds to $k(t)$. In $4 \mathrm{G}$ and $5 \mathrm{G}$ systems, the modulated symbols are loaded onto frequencydomain subcarriers that constitute an orthogonal frequency division multiplexing (OFDM) symbol. Several, contiguous, OFDM symbols are packed into a frame along with known reference symbols for channel estimation and transmitted over the air. The effective data transmission rate is

$$
r_{k}=\frac{1}{\Delta f \Delta t} D_{k} \quad[\text { bits / s / Hz }],
$$

for MCS index $k(t)=k$, where $\Delta f$ and $\Delta t$ denote the bandwidth and time spanned by the frame respectively.

The complex-valued and time-varying wireless channel, denoted here by $\mathbf{h}_{t}$, attenuates the transmitted signal and adds stochastic impairments such as phase rotation, noise, and interference. The receiver estimates the channel $\hat{\mathbf{h}}_{t}$ from the reference symbols embedded in the frame and uses it to compensate for channel effects on the information-containing symbols. The receiver then inverses the data processing steps applied prior to transmission and constructs the received transport block. The successful reception of transport block is validated using a cyclic redundancy check of the recovered bits, and an $\operatorname{ACK}\left(x_{k(t)}=1\right)$ or NACK $\left(x_{k(t)}=0\right)$ signal indicating respective success or failure is fed back to the base station. Additionally, the receiver periodically estimates a discrete CQI, $q_{t}=f\left(\hat{\mathbf{h}}_{t}\right) \in \mathcal{Q}$, which is fed back to base station. For a choice of MCS indices $k(t)=k \in 1, \ldots, K$ and observed CQI $q(t)=q \in \mathcal{Q}$, the binary transmission success events $x_{k, q}=x\left(r_{k}, q\right)$ can be viewed as a family of stochastic random variables, which yields the conditional transmission success probability $\mu_{k, q}=P\left(x_{k, q}=1 \mid q\right)$. We model this probability as an independent and identically distributed Bernoulli random variable, i.e., $x_{k, q} \sim \operatorname{Bern}\left(\mu_{k, q}\right)$.

\section{B. Optimal MCS Selection under a BLER Target}

Consider a wireless link with true transmission success probabilities $\mu_{k}, k \in\{1, \ldots, K\}$ for the corresponding set of available rates $r_{k}$ and for a given CQI. In each frame indexed by the time interval $t=1, \ldots, T$, selecting the MCS index $k(t) \in\{1, \ldots, K\}$ either delivers $r_{k(t)}$ bits to the receiver with probability $\mu_{k(t)}$, or zero bits with probability $1-\mu_{k(t)}$. Under the assumption that CQI captures the wireless channel state accurately, the packet success event is a Bernoulli random variable with mean $\mu_{k}$, i.e., $x_{k} \sim \operatorname{Bern}\left(\mu_{k}\right)$. The goal of link adaptation is to maximize the average throughput over $T$ frames subject to the BLER target $\eta$,

$$
\begin{array}{ll}
\operatorname{maximize} & \frac{1}{T} \sum_{t=1}^{T} r_{k(t)} x_{k(t)} \\
\text { subject to } & \frac{1}{T} \sum_{t=1}^{T} x_{k(t)} \geq 1-\eta
\end{array}
$$

Denote by $n_{k}(T)$ and $s_{k}(T)$ the number of times that MCS index $k(t)=k$ is selected and the number of observed successes respectively until frame $T$. Assuming that the transmission success events are independent and identically distributed, we have the optimal MCS selection problem:

$$
\begin{aligned}
& \underset{n_{1}(T), \ldots, n_{K}(T)}{\operatorname{argmax}} \frac{1}{T} \sum_{k=1}^{K} r_{k} n_{k}(T) s_{k}(T) \\
& \quad \text { subject to } \frac{1}{T} \sum_{k=1}^{K} n_{k}(T) s_{k}(T) \geq 1-\eta .
\end{aligned}
$$

The optimal solution to (3) is NP-hard and requires computational time that is exponential in $T$. However, wireless applications are designed to be tolerant to occasional transmission failures due to unpredictable radio channel conditions. This implies that the BLER target constraint in (3) can be transformed to a soft constraint that needs to be satisfied only in the expectation sense. Recall that the packet success probability for rate $r_{k}$ is denoted by $\mu_{k}$, then we can write the relaxation of (3) as the linear program

$$
\begin{aligned}
L P(\boldsymbol{p}):= & \operatorname{maximize} \sum_{k=1}^{K} p_{k} r_{k} \mu_{k} \\
& \text { subject to } \sum_{k=1}^{K} p_{k} \mu_{k} \geq 1-\eta,
\end{aligned}
$$

where $p_{k} \in[0,1]$ is the probability of selecting MCS index $k$, and $\boldsymbol{p}=\left[p_{1}, \ldots, p_{K}\right]$ is the probabilistic MCS selection vector such that $\sum_{k=1}^{K} p_{k}=1$. We denote the stationary optimal solution to (4) by $\boldsymbol{p}^{*}=\left[p_{1}^{*}, \ldots, p_{K}^{*}\right]$.

The problem in (4) is an optimization problem with a linear objective and a linear constraint. The space of the feasible objective values, $\sum_{k=1}^{K} p_{k} r_{k} \mu_{k}$ is the convex hull of the expected throughputs, $r_{k} \mu_{k}, k \in\{1, \ldots, K\}$. There are three possible scenarios: First, no feasible solution to (4) exists. Second, an optimal solution exists and is equivalent to always 
selecting a certain MCS for transmission. Third, an optimal solution exists that is composed of a linear combination of more than one available MCSs. We describe these three scenarios subsequently.

Proposition 1. For a set of transmission success probabilities $\left\{\mu_{1}, \ldots, \mu_{K}\right\}$ such that $\mu_{k}<1-\eta \forall k \in\{1, \ldots, K\}$, there does not exist any feasible solution to (4).

Proof. In this case, we have that $\sum_{k=1}^{K} \mu_{k} p_{k} \leq$ $\max _{k} \mu_{k} \sum_{k=1}^{K} p_{k} \leq \max _{k} \mu_{k}<1-\eta$. Therefore, the BLER target constraint cannot be satisfied using the available MCSs. Consequently, no feasible solution to the problem exists.

Next, we describe the scenarios under which a feasible solution exists. In the following, we assume that the rate corresponding to the MCS index $i=\operatorname{argmax}_{k \in\{1, \ldots, K\}} r_{k} \mu_{k}$ is the throughput-maximizing rate. Then we consider the two non-overlapping scenarios where the MCS index $i$ satisfies and fails the BLER target respectively, i.e., $\mu_{i} \geq 1-\eta$ and $\mu_{i}<1-\eta$ respectively.

Proposition 2. If $\mu_{i} \geq 1-\eta$, the optimal rate selection probabilities are degenerate to $p_{k}^{*}=1, k=i$ and $p_{k}^{*}=0 \forall k \neq i$.

Proof. We have that $\sum_{k=1}^{K} r_{k} \mu_{k} p_{k} \leq r_{i} \mu_{i} \sum_{k=1}^{K} p_{k} \leq r_{i} \mu_{i}$ with equality iff $p_{i}=1$. Thus, always selecting the MCS index $i$ maximizes the objective. Further, since $\sum_{k=1}^{K} \mu_{k} p_{k}=\mu_{i} \geq$ $1-\eta$, the BLER constraint is satisfied for this MCS selection scheme. Therefore, the optimal MCS selection probabilities are degenerate to $p_{k}=1, k=i$ and $p_{k}=0 \forall k \neq i$.

Proposition 3. If $\mu_{i}<\beta$ and a feasible solution exists, then the probabilistic MCS selection vector is supported by at least two MCS indices.

Proof. Consider the set of arms $\mathcal{U}^{\prime}:=\left\{i \mid r_{i} \mu_{i}>\right.$ $\left.\max _{k \in \mathcal{U}} r_{k} \mu_{k}, \mu_{k}<1-\eta\right\}$. Then for any $k_{1} \in \mathcal{U}^{\prime}$ and $k_{2} \in \mathcal{U}$, and $\epsilon>0$, there exists another constant $\delta>0$ such that $\epsilon \mu_{k_{1}}+\delta \mu_{k_{2}}=(\epsilon+\delta)(1-\eta)$. Since $\mu_{k_{1}} r_{k_{1}}>\mu_{k_{2}} r_{k_{2}}$ by definition, we have that $\epsilon \mu_{k_{1}} r_{k_{1}}+\delta \mu_{k_{2}} r_{k_{2}}>(a+b) \mu_{k_{2}} r_{k_{2}}$. Setting $\epsilon+\delta=p_{k_{2}}$, where $p_{k_{2}}$ is the contribution of the MCS index $k_{2}$ towards the success probability, we obtain $\epsilon$ and $\delta$ such that $\epsilon \mu_{k_{1}}+\delta \mu_{k_{2}}=p_{k_{2}}(1-\eta)$ and $\epsilon \mu_{k_{1}} r_{k_{1}}+$ $\delta \mu_{k_{2}} r_{k_{2}}>p_{k_{2}} \mu_{k_{2}} r_{k_{2}}$. Repeating this process for all $k \in \mathcal{U}$, we obtain the optimal MCS selection vector that satisfies this proposition. Consequently, the optimal link adaptation policy is a probabilistic mixture of more than one MCS indices.

Hence, for any given CQI, the optimal MCS selection strategy corresponding to (4) has three possibile outcomes: (i) no feasible solution, (ii) the throughput-maximizing MCS also satisfies the constraint and therefore is the single optimal MCS, and (iii) a probabilistic mixture of two MCSs optimizes the throughput subject to the BLER target. In the rest of this paper, we propose BayesLA, a Bayesian scheme that efficiently learns the optimal MCS selection strategy online, and present numerical performance results.

\section{LINK ADAPTATION SCHEMES}

\section{A. Outer Loop Link Adaptation}

The base station obtains periodic, discretized CQI values, $q \in \mathcal{Q}$, from the receiver. In OLLA, the transmitter maps the CQI to approximate transmission success probabilities, $\bar{\mu}_{1, \ldots, K}$, using a parameterized, offline link-to-system (L2S) lookup table $\mathcal{M}_{\theta}(q): q \mapsto \bar{\mu}_{1, \ldots, K}$ [11]. These transmission success probabilities are corrupted by modeling errors, quantization noise, and feedback delays. The transmitter compensates for these inaccuracies through a dynamic offset applied to the the parameters of the L2S model through OLLA. OLLA adds a fixed offset, $\Delta^{\text {up }}=\Delta$, for every observed transmission success and subtracts another, fixed, offset, $\Delta^{\text {down }}$, to the model parameters for for every observed transmission failure. The ratio of offset values are chosen to be $\frac{\Delta^{\text {up }}}{\Delta^{\text {down }}}=\frac{1-\eta}{\eta}$ such that OLLA converges asymptotically to the BLER target $\eta$. In every frame $t$, OLLA uses the latest parameter offset, $\Delta_{t}^{\text {OLLA }}$, to estimate the transmission success probabilities for each MCS through a mapping function $f$ over the local offline model. The OLLA algorithm steps are described in Alg. 1.

There are three major shortcoming of OLLA: (i) OLLA converges to the BLER target $\eta$. However, as discussed in Sec. II-B, the optimal BLER target is not known analytically. As a results, the MCS selected by OLLA after convergence may provide a sub-optimal throughput. (ii) The steady-state performance of OLLA depends on the scaling factor $\Delta$ for the step adjustments. For relatively large step sizes, OLLA converges quickly towards the optimal rate selection policy, but suffers from larger fluctuations around the steady state. On the other hand for small step sizes, OLLA has stable steady state performance at the cost of slower convergence. (iii) Finally from the definition of step sizes, we observe that OLLA convergence is a function of $\eta$. For relatively large values of $\eta$, the upward adjustment step is small, and conversely for larger values of $\eta$, the downward adjustment step is small.

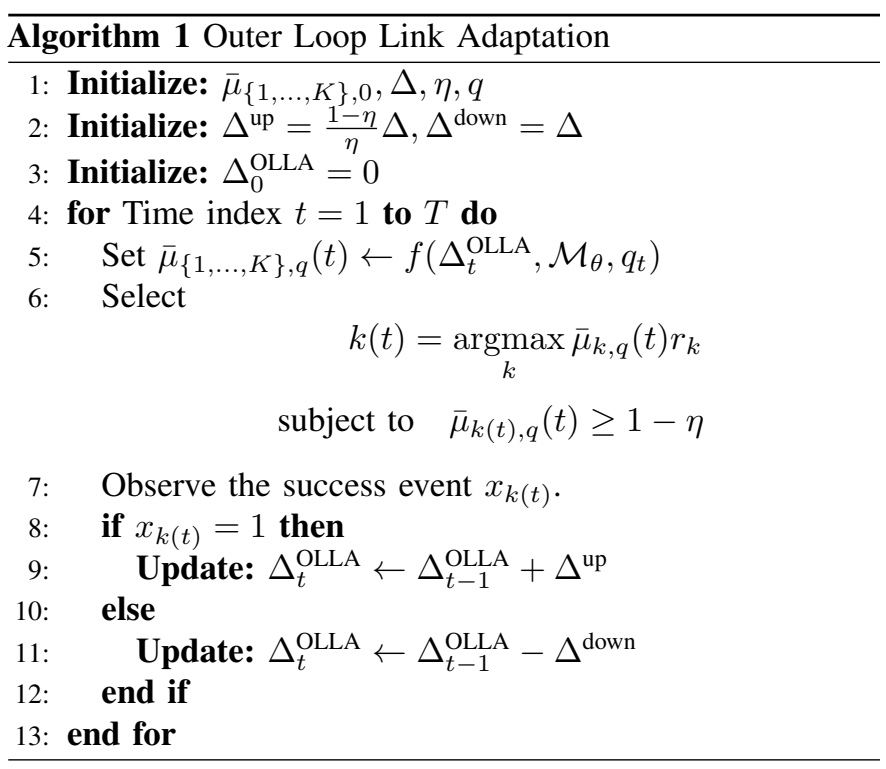




\section{B. BayesLA}

The shortcomings of OLLA arise from the fact that it depends on a careful choice of tuning parameters for optimality. In contrast, we propose BayesLA, a Bayesian link adaptation approach, where the algorithm parameters are learnt automatically from the observed ACK / NACKs for prior transmissions. BayesLA first assigns uniform prior distributions over a priori unknown conditional transmission success probabilities, $\mu_{k, q}$. In each frame, BayesLA samples these distributions to estimate the MCS that maximizes the expected throughput under the BLER target. Subsequently, BayesLA refines the posterior distributions with the observed ACK / NACK value for the selected MCS. This sampling-based approach, also known as Thompson Sampling, efficiently balances the explorationexploitation trade-off in learning the optimal action from a set of finite available actions [8].

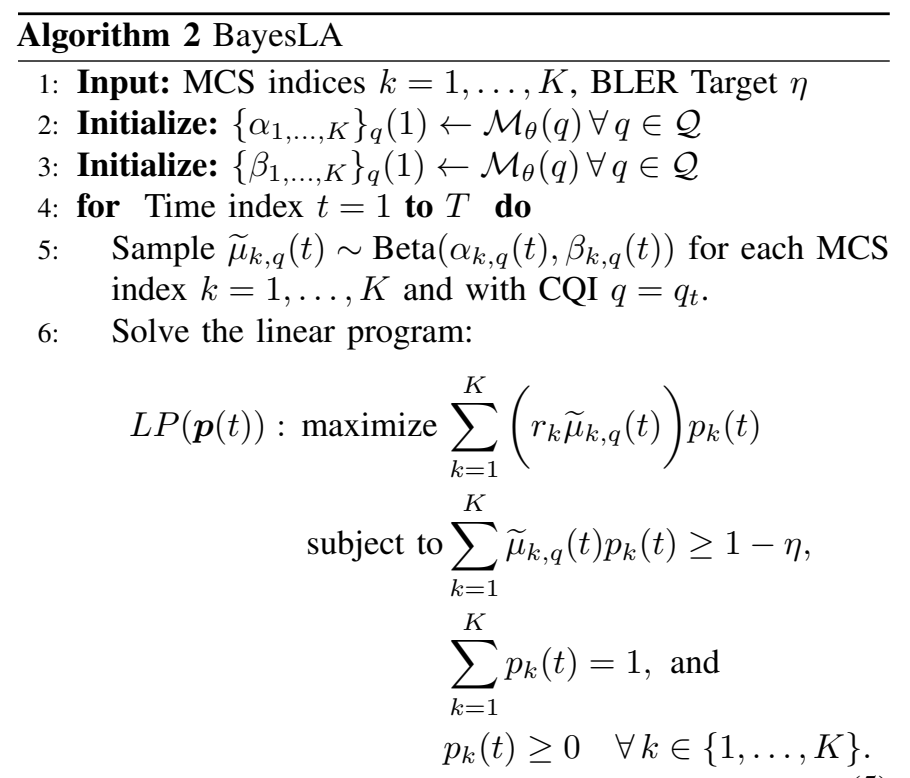

7: if Eq. 5 has a feasible solution, then

8: $\quad$ Sample $k(t) \sim\left[p_{1}^{*}(t), \ldots, p_{K}^{*}(t)\right]$

9: $\quad$ else

10: $\quad$ Sample $k(t)$ uniformly from $\{1, \ldots, K\}$.

11: end if

12: Observe: Transmission success $x_{k(t)}$.

13: Update:

$$
\begin{aligned}
& \alpha_{k(t), q}(t+1)=\alpha_{k(t), q}(t)+x_{k(t)} \\
& \beta_{k(t), q}(t+1)=\beta_{k(t), q}(t)+\left(1-x_{k(t)}\right) .
\end{aligned}
$$

14: end for

BayesLA assigns independent Beta priors, $\operatorname{Beta}\left(\alpha_{k, q}(1), \beta_{k, q}(1)\right)$, to the mean transmission success probability for each combination of MCS and CQI values using the offline L2S model $\mathcal{M}_{\theta}(q)$. These priors are updated with the observed ACK / NACK for the transmitted MCS, and the updated distributions are used for MCS selection in the next frame. The choice of Beta priors follows naturally from the conjugacy property of Beta and Bernoulli distributions. In particular, since the observed ACK, $x_{k(t), q}=1$, is Bernoulli-distributed (Sec. II-A), the posterior distribution over $\mu_{k(t), q}$ is simply another Beta-distributed random variable with parameters $\left(\alpha_{k(t), q}(t)+1, \beta_{k(t), q}(t)\right)$. Conversely for an observed NACK, the posterior Beta distribution has the parameters $\left(\alpha_{k(t), q}(t), \beta_{k(t), q}(t)+1\right)$.

In each frame indexed by the time interval $t$, BayesLA samples the success probabilities, $\widetilde{\mu}_{k, q}(t), k \in\{1, \ldots, K\}$, from the latest posterior for each MCS and for the observed CQI value. The estimated success probabilities are used to solve the LP in (5) and obtain the probabilistic MCS selection vector $\left[p_{1}^{*}(t), \ldots, p_{K}^{*}(t)\right]$. The optimal MCS index, $k(t)$ is obtained by sampling this vector. For the selected MCS, the transmission success or failure event, ACK / NACK, is observed through the binary variable $x_{k(t)}$, which is used to update the Beta distributions for each MCS according to the update rule in (6).

Compared to the empirically-selected OLLA parameters, the BayesLA algorithm parameters $\alpha_{1}, \ldots, \alpha_{K}, \beta_{1}, \ldots, \beta_{K}$ are initialized using the L2S model and refined online from the observed ACK / NACK for the transmitted MCSs. In [7], it was shown that the theoretical convergence of a closely related algorithm is guaranteed to be sub-linear in time. No comparable results are available for OLLA. Further, since BayesLA solves the sampled $L P(\boldsymbol{p}(t))$ in every frame, it obtains the optimal probabilistic MCS selection strategy. Hence, BayesLA does not suffer from the problem of sub-optimally defined BLER target as in the case of OLLA.

\section{Numerical EVALUATION}

We conduct numerical simulations for a $4 \mathrm{G}$ wireless link models in Python. The transmission parameters are obtained from the $4 \mathrm{G}$ physical-layer standard [12] and are listed in Tab. I. To obtain the offline L2S model, $\mathcal{M}_{\theta}(q)$ use the approach in [11]. The offline model is used to aid in link adaptation over a single-tap, Rayleigh fading channel with a normalized Doppler of 0.01. For each experiment, we simulate a downlink data transmission channel with 5000 contiguous frame transmissions. The throughput and block error rates are obtained by averaging the realized throughput and the NACKs respectively over 1000 independent links with randomly generated channel and noise realizations. We simulate two algorithms: OLLA, and BayesLA respectively. The OLLA step size is chosen to be $0.1 \mathrm{~dB}$ based on the analysis in [2]. We initialize the priors for BayesLA in the following manner: For the $k^{\text {th }}$ MCS, the prior is Beta $(1+$ $\left.\left\lfloor N \bar{\mu}_{k}(q)\right\rfloor, 1+\left\lfloor N\left(1-\bar{\mu}_{k}(q)\right)\right\rfloor\right)$, where $\bar{\mu}_{k}(q)$ is the success probability provided by the L2S model $\mathcal{M}_{\theta}(q)$ for the channel quality index $q$ and $N=10$ controls the confidence in the L2S model. The complete simulation code is available at [13].

In Fig. 1(a) and Fig. 1(b), we plot the realized throughput and the realized BLER for the considered algorithms, when the BLER target is set to 0.1. The BayesLA and OLLA algorithms have similar throughput at the beginning of the experiment along with a BLER close to 0.1. However, after a few transmissions, BayesLA achieves a slightly better throughput than OLLA. In addition, BayesLA converges to a lower BLER 


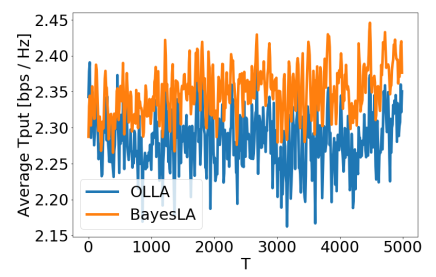

(a) Throughput for BLER Target 0.1

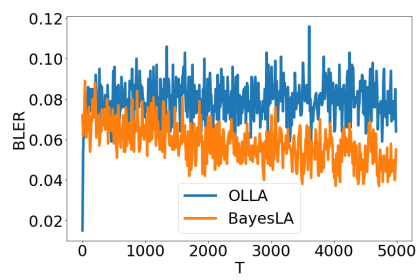

(b) BLER for BLER Target 0.1

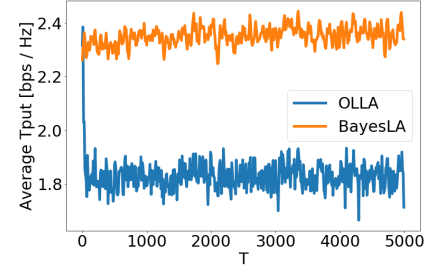

(c) Throughput for BLER Target 0.3

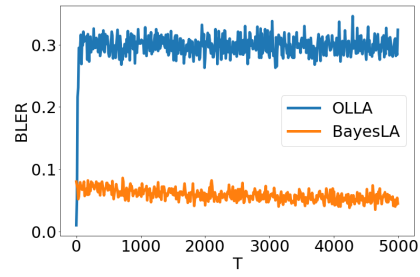

(d) BLER for BLER Target 0.3

Fig. 1. Average throughput and BLER performance for outer loop link adaptation (OLLA) and Bayesian Link Adaptation (BayesLA) for a Rayleigh fading channel. BayesLA achieves a higher throughput and lower BLER compared to OLLA, thereby improving the link performance.

TABLE I

SIMULATION PARAMETERS

\begin{tabular}{ll}
\hline Parameter & Value \\
\hline$K$ & 28 \\
$T$ & 1000 \\
$\eta$ & $0.1,0.3$ \\
SNR & $15 \mathrm{~dB}$ \\
Channel fading type & Rayleigh \\
Normalized Doppler & 0.01 \\
Number of subcarriers & 72 (i.e., 6 resource blocks) [12] \\
Transport Block Sizes & From [12, Tab. 7.1.7.2.1-1] \\
Modulation orders & From [12, Tab. 7.1.7.1-1] \\
\hline
\end{tabular}

compared to OLLA. Hence, by training the BayesLA priors on the offline L2S model, it is possible to achieve a good initial performance, and subsequently refine this further through online parameter updates to improve the performance compared to state-of-the-art OLLA.

In Fig. 1(c) and Fig. 1(d), we plot the realized throughput and the realized BLER for a BLER target of 0.3. For this BLER target, we find that OLLA suffers a significant performance drop. Since OLLA is designed to converge to the BLER target, it adjusts its offset values such that it converges to a BLER close to 0.3. However, since a BLER target of 0.3 is sub-optimal for this particular wireless configuration, the throughput performance of OLLA is sub-optimal. In contrast, BayesLA optimizes the throughput under the BLER target of 0.3 , thereby achieving a high throughput along with a lower BLER value, which is optimal for the simulated wireless environment scenario. Hence, BayesLA mitigates the effect of poorly defined link adaptation parameters.

\section{CONCLUSiOnS}

In this paper, we address link adaptation in terms of MCS selection under BLER target. We formulate a rigorous model of this constrained optimization problem and develop a linear programming relaxation suitable for the wireless setting. We propose a Bayesian, online learning algorithm, BayesLA, which optimizes the link throughput by learning the optimal probabilistic MCS selection policy. Further, compared to the state-of-the-art OLLA approach, BayesLA mitigates the reliance on heuristically-configured parameters that are ofted difficult to tune for optimal link performance. Through numerical experiments, we demonstrate that BayesLA improves the link performance compared to OLLA both in terms of the realized throughput and BLER.

\section{ACKNOWLEDGEMENTS}

This work was partially supported by the Wallenberg Artificial Intelligence, Autonomous Systems and Software Program (WASP) funded by Knut and Alice Wallenberg Foundation, and by the European Research Council project AGNOSTIC (742648).

\section{REFERENCES}

[1] A. Molisch, Wireless Communications. Wiley, 2010.

[2] P. Wu and N. Jindal, "Coding versus arq in fading channels: How reliable should the phy be?," IEEE Transactions on Communications, vol. 59, no. 12, pp. 3363-3374, 2011.

[3] S. Park, R. C. Daniels, and R. W. Heath, "Optimizing the target error rate for link adaptation," in 2015 IEEE Global Communications Conference (GLOBECOM), pp. 1-6, IEEE, 2015.

[4] V. Buenestado, J. M. Ruiz-Avilés, M. Toril, S. Luna-Ramírez, and A. Mendo, "Analysis of throughput performance statistics for benchmarking lte networks," IEEE Communications Letters, vol. 18, pp. 1607-1610, Sept 2014.

[5] V. Saxena, J. Jaldén, M. Bengtsson, and H. Tullberg, "Deep learning for frame error probability prediction in bicm-ofdm systems," in 2018 IEEE International Conference on Acoustics, Speech and Signal Processing (ICASSP), pp. 6658-6662, IEEE, 2018.

[6] R. A. Delgado, K. Lau, R. Middleton, R. S. Karlsson, T. Wigren, and Y. Sun, "Fast convergence outer loop link adaptation with infrequent updates in steady state," in 2017 IEEE 86th Vehicular Technology Conference (VTC-Fall), pp. 1-5, Sept 2017.

[7] V. Saxena, J. Jaldén, J. E. Gonzalez, M. Bengtsson, H. Tullberg, and I. Stoica, "Contextual multi-armed bandits for link adaptation in cellular networks," in Proceedings of the 2019 Workshop on Network Meets AI \& $M L$, pp. 44-49, 2019.

[8] O. Chapelle and L. Li, "An empirical evaluation of thompson sampling," in Advances in neural information processing systems, pp. 2249-2257, 2011.

[9] H. Qi, Z. Hu, X. Wen, and Z. Lu, "Rate adaptation with thompson sampling in 802.11 ac wlan," IEEE Communications Letters, vol. 23 no. 10 , pp. $1888-1892,2019$.

[10] V. Saxena, J. Jaldén, J. E. Gonzalez, I. Stoica, and H. Tullberg, "Constrained thompson sampling for wireless link optimization," arXiv preprint arXiv:1902.11102, 2019.

[11] K. Brueninghaus, D. Astely, T. Salzer, S. Visuri, A. Alexiou, S. Karger, and G.-A. Seraji, "Link performance models for system level simulations of broadband radio access systems," in Personal, Indoor and Mobile Radio Communications, 2005. PIMRC 2005. IEEE 16th International Symposium on, vol. 4, pp. 2306-2311, IEEE, 2005.

[12] 3rd Generation Partnership Project, "Evolved Universal Terrestrial Radio Access (E-UTRA); Physical layer procedures," Tech. Rep. 36.213 v12.3.0, Sept. 2016.

[13] V. Saxena, "bayesla-link-adaptation." https://github.com/vidits-kth/ bayesla-link-adaptation, 2020. 